\title{
Instrumentos para Avaliação da Capacidade Parental e Familiar em Situações de Risco
}

\author{
Ana Teixeira de Melo ${ }^{1}$ \\ Madalena Alarcão \\ Universidade de Coimbra
}

\begin{abstract}
RESUMO - O Modelo de Avaliação e de Intervenção Familiar Integrada (MAIFI) incorpora um processo de avaliação que visa informar a tomada de decisão em situações de risco psicossocial ou de perigo para a criança, bem como a elaboração de projetos de apoio à família. Os Instrumentos de Síntese da Avaliação Compreensiva no MAIFI (ISACM) foram construídos para apoiar a organização e síntese desse processo de avaliação. O objetivo deste estudo é avaliar as suas propriedades psicométricas no que diz respeito à validade de construto, validade concorrente e fidedignidade por avaliação da consistência interna das subescalas e acordo entre avaliadores. Os ISACM revelaram propriedades psicométricas bastante satisfatórias que permitem recomendar a sua utilização na organização e síntese de avaliações compreensivas.
\end{abstract}

Palavras-chave: avaliação familiar, avaliação do risco, crianças em risco, capacidade parental, proteção da criança

\section{Instruments for the Assessment of Parental and Family Capacity in Risk Situations}

\begin{abstract}
The Integrated Family Assessment and Intervention Model (IFAIM) incorporates an assessment process which aims to inform decision making in situations of psychosocial risk or danger for the child, as well as the elaboration or family support projects. The Instruments of Synthesis of Comprehensive Assessments in IFAIM (ISCAI) were built to support the organization and synthesis of this assessment process. The purpose of this study is to evaluate its psychometric properties concerning construct validity, concurrent validity and reliability, considering internal consistency and inter-rater agreement. The ISCAI revealed quite satisfactory psychometric properties which allow recommending its use in the organization and synthesis of comprehensive assessments.
\end{abstract}

Keywords: family assessment, risk assessment, at-risk children, parental capacity, child protection

Nas últimas décadas, e apesar das limitações, a investigação foi acumulando conhecimento sobre os mecanismos e fatores de risco e proteção implicados na construção de trajetórias desenvolvimentais adaptativas ou perturbadas das crianças (Cicchetti \& Cohen, 2006; Masten, 2007). Neste enquadramento, a família é percebida como uma fonte de influência próxima da criança e entendida como um nível importante de análise na compreensão ecológica das situações de maus tratos e negligência (Belsky, 1980; Cicchetti \& Toth, 1997, Cicchetti \& Valentino, 2006).

O progressivo reconhecimento, por parte das sociedades atuais, do dever de proteção da criança faz-se acompanhar não só de esforços da comunidade científica para identificar condições associadas ao aparecimento de maus tratos e negligência mas, também, do desenvolvimento de instrumentos para estimação do risco de (re)ocorrência dos mesmos (DePanfilis, 1996; Wald \& Woolverton, 1990). Alguns sistemas sociais e de proteção da criança têm sentido a necessidade de oferecer a todas as famílias uma avaliação compreensiva, isto é, que permita identificar as suas necessidades e prover serviços para satisfazê-las de forma adequada (Department of Health, 2000; Wise, 2003), particularmente nas situações de famílias com necessidades múltiplas e complexas (Spratt \& Devaney, 2009).

1 Endereço para correspondência: Ana Teixeira de Melo, Apartado 1562, EC Devesas, Vila Nova de Gaia, Portugal. CEP: 4400. E-mail: anamelopsi@gmail.com
Consideramos como sendo uma avaliação compreensiva integradora aquela que procura captar a complexidade das dinâmicas que sustentam o desenvolvimento da família e da criança, dos mecanismos que podem constranger ou potenciar o seu desenvolvimento, bem como das suas forças e vulnerabilidades (Johnson et al., 2006; Schene, 2005). Por conseguinte, tende a incluir informação sobre fatores de risco e de proteção ao nível do funcionamento da família, como um todo, do desenvolvimento dos prestadores de cuidados e capacidade parental e do percurso ontológico da própria criança, para além das condições ambientais, sociais e culturais que os enquadram (Corcoran \& Nichols-Casebolt, 2004; Farnfield, 2008; Fowler, 2003; Jack, 2001; Rose, 2001). Estas avaliações devem, assim, constituir a base de trabalho dos serviços centrados na família porque oferecem indicações sobre as dimensões a considerar na elaboração de projetos de suporte para a mudança.

Ao contrário do que acontece em outros países (Lévleillé \& Chamberland, 2010), em Portugal existem poucos modelos gerais de avaliação e instrumentos específicos que orientem os profissionais na coleta, organização, síntese e análise da informação. Na realidade, as Comissões de Proteção de Crianças e Jovens parecem apresentar insuficientes competências técnicas para conduzir avaliações e diferenciar situações de risco de casos de perigo (Torres, 2008), bem como lacunas formativas ao nível das metodologias de trabalho ([CNPCJP], 2009). Antecipa-se que estas 
dificuldades sejam partilhadas também por outros serviços sociais e de apoio à família.

O Modelo de Avaliação e de Intervenção Familiar Integrada (MAIFI) tem vindo a ser recentemente implementado em Portugal, em Centros de Apoio Familiar e de Aconselhamento Parental (CAFAP) que prestam apoio a famílias multidesafiadas com crianças e jovens em situações de risco psicossocial, maltratadas ou negligenciadas. $\mathrm{O}$ modelo serve, ainda, o sistema de proteção da criança e outros serviços sociais na medida em que conduz avaliações do risco e avaliações do potencial de mudança das famílias. O MAIFI prescreve um processo para a condução destas avaliações, oferecendo aos profissionais um conjunto de instrumentos que orientam não só a recolha de informação como a síntese e análise da mesma. Estes instrumentos podem, contudo, vir a ser utilizados por profissionais de outros serviços sociais ou de proteção da criança que, no decurso do seu trabalho, se confrontam com famílias com crianças e jovens em situação de risco ou perigo e, por conseguinte, com um conjunto de decisões sobre o tipo de ação mais apropriado (Darlington, Healy, \& Feeney, 2010). Pela centralidade que assumem na condução das avaliações compreensivas, no MAIFI, e pelo seu potencial contributo para uma melhor articulação interinstitucional em matéria de avaliação em situações de risco e perigo, estes instrumentos necessitam da aferição das suas propriedades psicométricas e real utilidade.

Os Instrumentos de Síntese da Avaliação Compreensiva no MAIFI (ISACM) permitem sintetizar os resultados da avaliação compreensiva e integram: (a) o Instrumento de Síntese da Avaliação da Capacidade Parental, (b) o Instrumento de Síntese da Avaliação das Dinâmicas Familiares e Fatores que Afetam a Capacidade Parental e uma (c) o Instrumento de Síntese da Avaliação de Fatores Ambientais e Sociais. Um quarto instrumento sintetiza a avaliação centrada na criança mas, devido ao fato de ter um procedimento distinto de cotação, não é alvo desta investigação.

O objetivo deste estudo é avaliar as propriedades psicométricas destes instrumentos considerando: (a) a validade de construto; (b) a fidelidade, em termos da consistência interna das escalas; (c) a fidelidade relativa ao acordo entre avaliadores; (d) a validade concorrente por associação com uma escala atuarial de avaliação do risco.

\section{Método}

\section{Participantes}

Participaram neste estudo setenta e seis famílias acompanhadas por serviços de apoio familiar (Centros de Apoio Familiar e de Aconselhamento Parental; Gabinetes de Apoio Familiar), por terem crianças consideradas numa situação de risco psicossocial ou de perigo. As famílias forneceram o seu consentimento informado escrito para participação neste estudo, confirmando compreenderem que a sua participação em nada afetaria o apoio que receberiam por parte das equipes. Os motivos primários de sinalização das famílias relacionam-se com negligência parental (30,3\%), violência ou conflitos conjugais $(13,2 \%)$, dificuldades no exercício da parentalidade $(11,8 \%)$, comportamentos de risco dos menores/problemas de comportamento $(9,2 \%)$, carência económica/dificuldades de integração social $(5,2 \%)$, existência de múltiplos problemas/desafios associados a indicadores de perigo $(6,6 \%)$ ou sem associação aos mesmos $(5,3 \%)$, maus tratos físicos ou psicológicos $(5,3 \%)$ e adaptação a transições de vida normativas e não normativas $(5,3 \%)$. Outros problemas diversos foram assinalados para os restantes casos $(7,8 \%)$. O número médio de elementos por agregado familiar é de 4,05 $(\operatorname{Min}=2 ; \operatorname{Max}=8)$, com um número médio de 2,22 filhos ( $\operatorname{Min}=1 ; \operatorname{Max}=6)$. A média de idades do filho mais novo da família é de 5,80 anos (Min = 0,08; Max =17), enquanto a idade média dos pais é de 38,19 $(\operatorname{Min}=18 ; \operatorname{Max}=56)$ e a das mães é de 33,67 $(\operatorname{Min}=19$; Max = 53). A maioria das famílias tem a configuração de uma família nuclear $(52,6 \%)$, enquanto $27,6 \%$ são famílias monoparentais e 19,7\% apresentam outras configurações. Sessenta e quatro por cento das famílias têm crianças com processos acompanhados por Comissões de Proteção de Crianças e Jovens ou por Equipas Multidisciplinares de Assessoria aos Tribunais e 42,1\% das famílias beneficiam de algum tipo de acompanhamento social no âmbito do trabalho de apoio realizado pelas equipas da Segurança Social, de Rendimento Social de Inserção ou ligadas a habitação social. Uma porcentagem menor tem algum elemento que beneficia de serviços de saúde mental $(21,1 \%)$, de reinserção social $(7,9 \%)$ ou de tratamento de toxicodependência/alcoolismo $(3,9 \%)$.

\section{Instrumentos}

Desenvolvimento e Descrição dos Instrumentos de Síntese da Avaliação Compreensiva no MAIFI

Os Instrumentos de Síntese da Avaliação Compreensiva no MAIFI (ISACM, Melo \& Alarcão, 2008) foram desenvolvidos com o objetivo de organizar e de sintetizar a informação recolhida no âmbito de processos de avaliação compreensiva de famílias multidesafiadas com crianças e jovens em situação de risco psicossocial ou de perigo (mau trato físico, psicológico e negligência). Mais concretamente, os ISACM foram concebidas com o objetivo de permitir a identificação de fatores de risco ou perigo para o desenvolvimento da criança mas também de fatores de proteção, forças e competências em diferentes dimensões i) da capacidade e competências parentais, ii) do funcionamento familiar e dos prestadores de cuidados e iii) das circunstâncias ambientais e sociais da vida da família.

Neste sentido, estes instrumentos foram construídos compreendendo duas Partes distintas: (a) Parte I, centrada na avaliação e quantificação das diferentes dimensões em avaliação, através de uma escala de Likert de cinco pontos e (b) Parte II, avaliação qualitativa, composta por perguntas abertas, mas que não é analisada neste artigo.

seleção do conteúdo e a construção dos itens, assentou numa revisão da literatura orientada por uma compreensão ecológica do desenvolvimento da criança e da parentalidade, dos maus tratos (particularmente físicos e psicológicos) e da negligência, bem como pelos contributos da psicopatologia 
do desenvolvimento para a compreensão dos fatores de risco e de proteção psicossociais e dos mecanismos de resiliência (e.g. Belsky, 1980; 1984; Cicchetti \& Lynch, 1995; Cicchetti \& Valentino, 2006; Cummings, Davies \& Campbell, 2000; Walsh, 2002). A revisão da literatura foi também orientada para a identificação de variáveis chave, habitualmente consideradas em sistemas e instrumentos diversos de avaliação compreensiva familiar (e.g. Johnson, et al., 2006).

Os Instrumentos de Síntese de Avaliação Compreensiva no MAIFI consistem em três instrumentos distintos, embora teoricamente relacionados (ver apêndice 1) e que a seguir se descrevem.

O Instrumento de Síntese da Avaliação da Capacidade Parental foi construído de modo a incluir itens relacionados com áreas centrais da capacidade/competências parentais (e.g. Azar, Lauretti, \& Loding, 1998; Budd, 2001; Jones, 2001). Incluiu-se o mesmo número de dimensões contempladas no modelo britânico da Framework for the Assessment of Children in Need and their Families (Department of Health, 2000; Jones 2001) relativas à capacidade parental para satisfazer as necessidades da criança em termos de: cuidados básicos ( 7 itens); segurança física e proteção face a outros (3 itens); segurança afetiva (3 itens); estimulação (3 itens); orientação e estabelecimento de limites ( 3 itens) e estabilidade ( 2 itens).

O Instrumento de Síntese da Avaliação das Dinâmicas Familiares e Fatores que Afetam a Capacidade Parental foi construído de modo a incluir fatores relacionados com o funcionamento familiar, particularmente dimensões associadas a processos de resiliência familiar (Walsh, 2002) tais como: crenças/narrativas/identidade familiar (e.g. identidade familiar positiva; otimismo e esperança) (2 itens); padrões interacionais/estrutura/organização da vida familiar (coesão e união) (2 itens); comunicação/flexibilidade/ capacidade de adaptação e resolução de problemas (2 itens). Incluiu, ainda, outros fatores que, não constituindo um indicador direto da capacidade parental, podem, contudo, afetar essa capacidade, tais como dimensões relacionadas com a qualidade da relação conjugal ( 2 itens) e com a história desenvolvimental (1 item) e com outras características dos prestadores de cuidados (e.g. Azar, Lauretti, \& Loding, 1998; Belsky, 1984; Budd, 2001; Department of Health, 2000; Fowler, 2003; Reder \& Lucey, 1995), tais como sua a autopercepção (1 item); a satisfação com a parentalidade (1 item); o abuso de substâncias (2 itens); a psicopatologia (1 item), os padrões de funcionamento individual (3 itens) e a motivação para a mudança (1 item).

O Instrumento de Síntese da Avaliação de Fatores Ambientais e Sociais foi construído de modo a incluir a avaliação de fatores relacionados com a qualidade da habitação (4 itens), com o emprego/recursos financeiros (3 itens), a qualidade da integração social da família e do ambiente comunitário (4 itens) e com a gestão financeira e doméstica (2 itens).

A formulação dos itens foi feita pela primeira autora e revista pela segunda. Foi selecionada uma escala de Likert de cinco pontos para servir de base à organização da avaliação e cotação de cada item, procurando respeitar-se um critério, o mais uniforme possível, para definição de cada ponto desta escala. Assim, as classificações de 1 e 2 correspondem a áreas de incompetência/vulnerabilidade/ mau funcionamento que, teoricamente, podem criar circunstâncias de perigo para a criança. A diferença entre o ponto 1 e 2 consiste, essencialmente, no fato de, no primeiro, os prestadores de cuidados impedirem a prestação de cuidados adequados à criança, ou negarem qualquer forma de ajuda externa, e, no ponto 2 , tal não acontecer. No polo oposto, as classificações de 4 e 5 correspondem a níveis de competência/força/proteção claros. Regra geral, o ponto 4 corresponde a situações de competência bastante satisfatória e frequente enquanto o 5 contempla não só situações em que a competência seria sempre observada ou completamente satisfatória como também situações em que os prestadores de cuidados apresentam capacidade de a desenvolver autonomamente. $\mathrm{O}$ ponto 3 representa situações em que há forças mas também vulnerabilidades, ora representando oscilações da competência ao longo do tempo, ora evidenciando algumas dimensões de competência ou força mas, simultaneamente, de vulnerabilidades. Este item corresponde, teoricamente, a fatores de risco psicossocial que, no momento, não estão associados a um perigo imediato para a criança. Foi adicionada uma categoria de classificação "Não Aplicável/Não sei" tendo em consideração que, dependendo do propósito da avaliação, alguns itens podem não ser particularmente relevantes (e por isso não aplicáveis) ou que o profissional pode não dispor da informação para responder.

Foi elaborado um manual com instruções para utilização e cotação dos ISACM pela primeira autora e revisto pela segunda. Este manual, bem como os ISACM foram analisados, numa fase inicial, por um conjunto de 16 profissionais que desenvolviam trabalho de avaliação e intervenção com famílias multidesafiadas com crianças em situação de risco psicossocial, maltratadas ou negligenciadas em serviços de apoio familiar. Os ISACM foram testados em algumas aplicações piloto por estes profissionais e pela primeira autora tendo em vista a avaliação da adequação dos itens e da formulação dos pontos de cotação. Em função desta avaliação alguns itens dos ISACM e as instruções de cotação foram revistos, principalmente no que diz respeito à definição mais precisa de regras de cotação para pontos mais próximos da escala (e.g., 1-2; 2-3; ou 4-5).

California Family Risk Assessment. O California Family Risk Assessment (CFRA) é uma das versões do instrumento de avaliação atuarial do risco desenvolvido pelo Children's Research Center, integrado no Structured Decision Making ${ }^{\circledR}$, implementada e testada no estado da Califórnia (Wagner \& Johnson, 1999). O instrumento orienta o profissional na estimativa do risco de negligência e outras formas de abuso, classificando as famílias como apresentando um risco baixo, moderado, elevado ou muito elevado, a partir da identificação de fatores de risco referidos na literatura e empiricamente sustentados. O CFRS apresenta duas escalas de dez itens, distintas, para estimativa do risco de negligência e de abuso e tem apresentado boa validade preditiva (Johnson, 2011; Johnson \& Wagner, 2003). Para este estudo, foi feita uma tradução e adaptação do instrumento para a população portuguesa realizada pelos autores. Foi utilizado um procedimento de tradução e retroversão pela primeira autora. Numa reunião online foram discutidas, com o Children's 
Research Center, as dúvidas relativas à adequação dos itens tendo em conta as características do sistema de proteção português e discutidas possíveis adaptações dos itens menos adequados. O instrumento não foi avaliado para a população portuguesa tendo em conta a sua validade preditiva. No entanto, para o presente estudo, obteve-se um valor médio de acordo entre-avaliadores Kappa de 0,89 para a escala da negligência e 0,80 para a escala do abuso.

\section{Procedimento}

Vinte e cinco profissionais (psicólogos, assistentes sociais e educadores sociais), que integram equipes de centros e gabinetes de apoio familiar e de centros comunitários, receberam 4 horas de formação com primeira autora para o preenchimento dos ISACM. A formação contemplou uma introdução ao racional orientador da construção dos ISACM. Foi esclarecida a natureza da informação necessária e oferecido um protocolo com sugestões de procedimentos de avaliação. Foi usada a descrição escrita de dois casos para os profissionais treinarem o preenchimento dos ISACM e esclarecerem as dúvidas e dificuldades que pudessem surgir.

Dezesseis destes profissionais participaram em meio dia de formação online, ministrada pelo Children's Research Center para preenchimento do CFRA. A formação compreendeu uma apresentação do racional do instrumento e o seu preenchimento a partir de exemplos de casos fornecidos na formação. Imediatamente após a formação online o grupo repetiu e discutiu o procedimento de preenchimento do instrumento para dois casos adicionais. Os mesmos exemplos foram utilizados posteriormente para formação dos profissionais que não participaram nesta reunião e foram realizadas sessões de revisão e ajustamento da cotação do instrumento com a primeira autora.

Terminado este período de formação, pares de profissionais, em diferentes equipes, conduziram avaliações integradoras compreensivas de famílias multidesafiadas, com crianças e jovens em situação de risco psicossocial ou famílias com crianças e jovens em situação de perigo, encaminhadas para os respetivos serviços de apoio familiar. Concluído o processo de avaliação, cada profissional preencheu, independentemente, os ISACM e o CFRS. Posteriormente, o par de profissionais discutiu as cotações realizadas tendo em vista a negociação, por consenso, de uma cotação final.

\section{Resultados}

Tendo em vista a avaliação da validade de construto dos ISACM foram calculadas análises fatoriais, de caráter exploratório, para os Instrumentos A, B e C, utilizando-se os valores obtidos por consenso na cotação final dos itens. Embora os dados não tenham apresentado uma distribuição normal, alguns autores consideram que a análise fatorial é relativamente robusta face a desvios à normalidade (Laros, 2005), particularmente se produzir comunalidades entre 0,40 e 0,70 (Costelo \& Osborne, 2005, conforme citado por Abbad, Queiroga, \& Zerbini, 2012) e cargas fatoriais superiores a 0,32 (Tabachnick \& Fidell, 2001 conforme citado por Abbad et al., 2012). Estes pressupostos foram cumpridos. Embora o tamanho da amostra seja limitado, considerou-se adequada a análise fatorial tendo em conta os valores médios da comunalidades por análise e os resultados dos testes de Kaiser-Meyer-Olkin, e de esfericidade de Bartlett, cujos resultados se reportam adiante.

Em todas as análises foi utilizada uma solução de componentes principais, seguida de rotação varimax. Foram retidos fatores com eigenvalues superiores a 1, após confirmação da adequação do critério através da análise do screeplot.

As Tabelas 1 a 3 apresentam a distribuição final dos itens por fator e as respetivas cargas de saturação, bem como a porcentagem de variância explicada e variância total. Nas mesmas tabelas são apresentados os resultados da avaliação da fidelidade das escalas. Para avaliação da consistência interna das escalas foi calculado, para cada fator/subescala a estatística alpha de Cronbach. Tendo em vista a avaliação da fidelidade relativa ao acordo entre avaliadores foi calculada a estatística Kappa, para cada item dos ISACM, com base nas cotações individuais dos profissionais de cada par.

Para a análise fatorial do Instrumento de Síntese da Avaliação da Capacidade Parental foi obtido um valor de Kaiser-Meyer-Olkin, de adequação da amostra de 0,79. $\mathrm{O}$ teste de esfericidade de Bartlett é estatisticamente significativo $[\chi 2(210, \mathrm{~N}=72)=823,52 ; \mathrm{p}<0,001]$ e o valor médio das comunalidades é 0,71 . Como se pode verificar na Tabela 1, na análise deste instrumento foram retidos seis fatores, com um número de itens a variar entre 7 , para 0 primeiro, e um item, para dois dos fatores. A estrutura obtida, e a distribuição dos itens, são teoricamente congruentes com a literatura, organizando-se os itens em diferentes dimensões de capacidade/competências parentais. Os itens retidos nos fatores de item único correspondem à capacidade parental de garantir a segurança física da criança e de assegurar proteção face a outros, enquanto o fator com maior número de itens agrega itens relacionados com a prestação de cuidados básicos às crianças. Os valores de consistência interna são satisfatórios variando entre 0,68 para o fator 3 (Estimulação), com 3 itens, e 0,88 para o fator 1 (Prestação de cuidados básicos) com 7 itens. O acordo entre avaliadores, ao nível dos itens é, na generalidade dos itens substancial (Landis $\&$ Koch, 1977), variando entre valores de kappa de 0,69 e 0,88 . Os valores de correlação item-total deste instrumento variam entre 0,44 para o fator 1 e 0,81 , também, para o fator 1. Os valores de correlação de Spearman entre os fatores variam entre 0,30 (entre o fator 4 e 5) e 0,66 (entre o fator 2 e 3). Os valores de correlação de Spearman entre os fatores e o instrumento total variam entre 0,67 (fator 5 e 6 ) e 0,90 (fator 1).

Para a análise fatorial relativa do Instrumento de Síntese da Avaliação das Dinâmicas Familiares e Fatores que Afetam a Capacidade Parental obteve-se um valor de KaiserMeyer-Olkin de 0,74. O teste de esfericidade de Bartlett é estatisticamente significativo $[\chi 2(153, \mathrm{~N}=76)=686,03 ; \mathrm{p}<$ $0,001]$ e o valor médio das comunalidades é 0,69 . Conforme se pode confirmar na tabela 2 , foi obtida uma estrutura de 5 fatores, correspondentes a 5 dimensões cuja consistência interna alpha de Cronbach variou entre 0,68 , para o fator 
5 (Facilitadores da Mudança), com dois itens, e 0,96 para o fator 4 (Relação Conjugal) com dois itens, também. Os diferentes fatores agregam itens relativos à relação conjugal (fator 4), funcionamento familiar (fator 2), características individuais dos prestadores de cuidados que podem contribuir para a capacidade parental (fator 3 ) e dimensões relativas a um funcionamento perturbado dos mesmos (fator 4). Há, ainda, um fator, a reunir itens que podem representar dimensões facilitadoras da mudança parental (fator 5). Ao nível dos itens, o acordo entre avaliadores é, igualmente substancial, com valores de kappa que variam entre 0,64 e
0,83 . Os valores de correlação item-total variam entre 0,43 para o fator 3 e 0,92 para o fator 4 . Os valores de correlação de Spearman entre os fatores variam entre 0,11 (entre o fator 5 e 4) e 0,55 (entre o fator 2 e 3). Os valores de correlação de Spearman entre os fatores e o instrumento total variam entre 0,42 (fator 4) e 0,74 (fator 1).

Finalmente, para a análise fatorial relativa ao Instrumento de Síntese da Avaliação de Fatores Ambientais e Sociais obteve-se um valor de Kaiser-Meyer-Olkin de 0,75. O teste de esfericidade de Bartlett é estatisticamente significativo $\left[\chi^{2}(78, \mathrm{~N}=76)=455,04 ; \mathrm{p}<0,001\right]$ e o valor médio das

Tabela 1. Resultado da análise fatorial final, cargas de saturação nos fatores, variância explicada, alpha de Cronbach e acordo entre avaliadores Kappa para o Instrumento de Avaliação da Capacidade Parental

\begin{tabular}{|c|c|c|c|c|c|c|c|}
\hline Item & $\begin{array}{c}\text { Fator 1 } \\
\text { Prestação } \\
\text { de cuidados } \\
\text { básicos }\end{array}$ & $\begin{array}{c}\text { Fator } 2 \\
\text { Segurança } \\
\text { afetiva e } \\
\text { supervisão }\end{array}$ & $\begin{array}{c}\text { Fator } 3 \\
\text { Estimulação }\end{array}$ & $\begin{array}{c}\text { Fator } 4 \\
\text { Orientação e } \\
\text { limites }\end{array}$ & $\begin{array}{l}\text { Fator } 5 \\
\text { Segurança } \\
\text { física }\end{array}$ & $\begin{array}{c}\text { Fator } 6 \\
\text { Proteção face } \\
\text { a outros }\end{array}$ & Kappa a) \\
\hline 1 & $\mathbf{0 , 8 3}$ & & & & & & 0,72 \\
\hline 2 & 0,72 & & & & 0,36 & & 0,72 \\
\hline 3 & 0,55 & & & 0,36 & 0,47 & & $0,89^{\text {b) }}$ \\
\hline 4 & 0,87 & & & & & & $0,87^{\mathrm{b})}$ \\
\hline 5 & 0,57 & & & & 0,55 & & $0,83^{\mathrm{b})}$ \\
\hline 6 & 0,77 & & & & & & 0,83 \\
\hline 7 & 0,56 & & & & & & 0,88 \\
\hline 8 & 0,40 & & & & 0,65 & & 0,78 \\
\hline 9 & & & & & & 0,91 & $0,86^{\text {b) }}$ \\
\hline 10 & & 0,65 & & & & & $0,89^{\text {b) }}$ \\
\hline 11 & & 0,79 & & & $-0,32$ & & $0,64^{\mathrm{b})}$ \\
\hline 12 & 0,33 & 0,70 & & & & & $0,85^{\text {b) }}$ \\
\hline 13 & & 0,79 & & & & & $0,81^{\mathrm{b})}$ \\
\hline 14 & & - & 0,66 & & 0,34 & & $0,68^{\mathrm{b})}$ \\
\hline 15 & & 0,64 & & & & & $0,75^{\mathrm{b})}$ \\
\hline 16 & 0,34 & 0,34 & & 0,53 & & & 0,80 \\
\hline 17 & & & & 0,87 & & & 0,71 \\
\hline 18 & & & 0,63 & 0,63 & & & 0,85 \\
\hline 19 & & & 0,70 & 0,36 & & & 0,81 \\
\hline 20 & & 0,72 & & & 0,39 & & $0,71^{\mathrm{b})}$ \\
\hline 21 & & & 0,83 & & & & 0,69 \\
\hline $\begin{array}{l}\text { Variância } \\
\text { explicada }\end{array}$ & $35,64 \%$ & $11,63 \%$ & $7,97 \%$ & $5,91 \%$ & $5,08 \%$ & $4,99 \%$ & \\
\hline$\alpha$ Cronbach & 0,88 & 0,86 & 0,68 & 0,72 & & & \\
\hline $\begin{array}{c}\text { Total da } \\
\text { variância } \\
\text { explicada }\end{array}$ & $71,22 \%$ & & & & & & \\
\hline
\end{tabular}

Notas. São apresentadas apenas as cargas de saturação superiores a 0,30. Os valores a negrito indicam o fator ao qual o item foi agregado na solução fatorial final adotada.

a) Para todos os valores de Kappa $\mathrm{p}<0,001$.

b) Para estes itens não foi possível calcular a estatística Kappa uma vez que não foi possível cumprir com o requisito de construir tabelas 2 vias simétricas para todos os valores da variável, para os dois avaliadores uma vez que alguns valores não foram igualmente observados para os dois avaliadores. São indicados os valores de correlação de Spearman. 
comunidades é 0,71 . Conforme se pode verificar na tabela 3 , foi obtida uma estrutura de 4 fatores que distingue os itens relativos à qualidade da habitação (fator 1), daqueles relativos à gestão doméstica (fator2), à qualidade da rede e da integração social da família (fator 3) e, ainda, à empregabilidade dos prestadores de cuidados (fator 4). O fator/dimensão relativo à rede e integração social é o único que apresenta um valor de consistência interna insatisfatório que não melhorava com a remoção de qualquer item $(\alpha$ Cronbach $=0,36)$. Os restantes fatores/dimensões apresentam valores de alpha de Cronbach a variar entre 0,60 (para o fator 4, com dois itens) e 0,90 (para o fator 1, com cinco itens). O acordo entre avaliadores ao nível dos itens é substancial, variando entre valores de kappa de 0,62 e 0,78. Os valores de correlação item-total variam entre 0,20 para o fator 3 e 0,88 para o fator 1 . Os valores de correlação de Spearman entre os fatores variam entre 0,20 (entre o fator 1 e 2) e 0,38 (entre o fator 2 e 3 ). Os valores de correlação de Spearman entre os fatores e o instrumento total variam entre 0,54 (fator 4) e 0,77 (fator 1).

Procurou efetuar-se uma análise preliminar da validade concorrente do Instrumento de Avaliação da Capacidade Parental, por comparação com a avaliação do risco de negligência e de abuso possibilitada pela CFRA. Assim, numa primeira fase, e para cada dimensão, procedeu-se à criação de uma variável correspondente à contagem de pontuações de perigo. Esta contagem baseia-se no número de itens que os instrumentos associam a situações de perigo, ou seja, à soma do número de itens que foram cotados com 1 ou 2. Analisou-se, então, a distribuição dos casos pelos

Tabela 2. Resultados da análise fatorial final, alpha de Cronbach e acordo entre avaliadores Kappa para o Instrumento de Avaliação das Dinâmicas Familiares e Fatores que Afetam a Capacidade Parental.

\begin{tabular}{|c|c|c|c|c|c|c|}
\hline Item & $\begin{array}{c}\text { Fator } 1 \\
\text { Perturbação no } \\
\text { funcionamento } \\
\text { dos prestadores } \\
\text { de cuidados } \\
\end{array}$ & $\begin{array}{c}\text { Fator } 2 \\
\text { Funcionamento } \\
\text { familiar }\end{array}$ & $\begin{array}{c}\text { Fator } 3 \\
\text { Contributos } \\
\text { pessoais dos } \\
\text { prestadores de } \\
\text { cuidados } \\
\end{array}$ & $\begin{array}{c}\text { Fator } 4 \text { Relação } \\
\text { conjugal }\end{array}$ & $\begin{array}{c}\text { Fator5 } \\
\text { Facilitadores } \\
\text { da mudança }\end{array}$ & Kappa a) \\
\hline 1 & & 0,71 & & & & $0,77 b)$ \\
\hline 2 & & 0,72 & 0,33 & & & 0,73 \\
\hline 3 & & 0,79 & & & & $0,76^{\mathrm{b})}$ \\
\hline 4 & & 0,65 & & & & $0,89^{\mathrm{b})}$ \\
\hline 5 & & 0,64 & & & & $0,64^{\mathrm{b})}$ \\
\hline 6 & & 0,36 & 0,51 & & & 0,72 \\
\hline 7 & & & & 0,95 & & 0,77 \\
\hline 8 & & & & 0,96 & & 0,67 \\
\hline 9 & & & 0,49 & & & 0,64 \\
\hline 10 & & & 0,72 & & & $0,63^{\mathrm{b})}$ \\
\hline 11 & & & 0,79 & & & $0,69^{b)}$ \\
\hline 12 & 0,75 & & & & & 0,83 \\
\hline 13 & 0,90 & & & & & $0,94^{\mathrm{b})}$ \\
\hline 14 & 0,70 & & & & & $0,95^{\text {b) }}$ \\
\hline 15 & & & & & 0,85 & $0,88^{\mathrm{b})}$ \\
\hline 16 & 0,51 & & 0,64 & & & $0,68^{\mathrm{b}}$ \\
\hline 17 & 0,88 & & & & & 0,83 \\
\hline 18 & & 0,41 & & & 0,69 & 0,79 b) \\
\hline $\begin{array}{l}\text { Variância } \\
\text { explicada }\end{array}$ & $29,71 \%$ & $14,52 \%$ & $11,82 \%$ & $7,55 \%$ & $5,59 \%$ & \\
\hline$\alpha$ Cronbach & 0,85 & 0,81 & 0,73 & 0,96 & 0,68 & \\
\hline $\begin{array}{c}\text { Total da } \\
\text { variância } \\
\text { explicada }\end{array}$ & $69,19 \%$ & & & & & \\
\hline
\end{tabular}

Notas. São apresentadas apenas as cargas de saturação superiores a 0,30. Os valores a negrito indicam o fator ao qual o item foi agregado na solução fatorial final adotada.

a) Para todos os valores de Kappa $p<0,001$.

b) Para estes itens não foi possível calcular a estatística Kappa uma vez que não foi possível cumprir com o requisito de construir tabelas 2 vias simétricas para todos os valores da variável, para os dois avaliadores uma vez que alguns valores não foram igualmente observados para os dois avaliadores. São indicados os valores de correlação de Spearman. 
diferentes níveis de risco em função da contagem do número de indicadores de perigo, conforme apresentado na tabela 4. Regra geral, verifica-se que, a maioria dos casos classificados com risco baixo ou moderado não apresenta pontuações de perigo, embora alguns casos apresentem um ou mais comportamento de perigo. No nível de risco elevado, para o abuso, continua-se, contudo, a verificar-se dispersão dos casos pelos diferentes níveis de contagem de indicadores de perigo.

Após esta primeira análise descritiva, analisou-se, ainda, a associação entre o número de comportamentos de perigo em cada dimensão e a categoria da avaliação do risco de negligência e de abuso (avaliado pelo CFRA), recorrendo-se ao teste do Chi-quadrado com um teste de significância exato, calculando-se a medida de associação phi de Cramer. Relativamente ao risco de negligência, verificaram-se associações significativas com a contagem de comportamentos de perigo, por ordem da magnitude da associação, para as dimensões de segurança afetiva e supervisão $[\chi 2(18, \mathrm{~N}=73)=79,34 ; \mathrm{p}=0,012 ; \Phi \mathrm{c}=0,60]$, orientação e limites $[\chi 2(9, \mathrm{~N}=73)=32,74 ; \mathrm{p}=0,005 ; \Phi \mathrm{c}$ $=0,39]$, estimulação $[\chi 2(9, \mathrm{~N}=73)=27,90 ; \mathrm{p}=0,029$;
$\Phi c=0,36]$, mas não para a segurança física $\left[\chi^{2}(3, \mathrm{~N}=\right.$ $73)=9,2 ; p=0,083 ; \Phi c=0,35]$, prestação de cuidados básicos $[\chi 2(15, \mathrm{~N}=73)=33,52 ; \mathrm{p}=0,065 ; \Phi \mathrm{c}=0,39]$ ou proteção face a outros $[\chi 2(3, N=73)=6,24 ; p=0,131$; Фc $=0,29]$. Relativamente ao risco de outras formas de abuso, verificaram-se associações positivas com a contagem de comportamentos de perigo para as dimensões de proteção face a outros $[\chi 2(3, N=72)=19,86 ; p=0,001 ; \Phi c=0,52]$, segurança afetiva e supervisão $[\chi 2(18, N=72)=39,02 ; p$ $=0,009 ; \Phi c=0,42]$, estimulação $[\chi 2(9, \mathrm{~N}=72)=28,58$; $\mathrm{p}=0,002 ; \Phi \mathrm{c}=0,36]$ e orientação e limites $[\chi 2(9, \mathrm{~N}=72)$ $=24,70 ; \mathrm{p}=0,007 ; \Phi \mathrm{c}=0,34]$ mas não com as dimensões de prestação de cuidados básicos $[\chi 2(15, \mathrm{~N}=72)=19,29$; $\mathrm{p}=0,205 ; \Phi \mathrm{c}=0,30]$ e segurança física $[\chi 2(3, \mathrm{~N}=72)=$ $4,61 ; \mathrm{p}=0,194 ; \Phi \mathrm{c}=0,25]$.

\section{Discussão}

Pretendia-se com este estudo proceder a uma primeira avaliação das propriedades psicométricas dos Instrumentos de Síntese da Avaliação Compreensiva no MAIFI (ISACM).

Tabela 3. Resultados da análise fatorial final, alpha de Cronbach e acordo entre avaliadores Kappa para o Instrumento de Avaliação de Fatores Ambientais e Sociais.

\begin{tabular}{|c|c|c|c|c|c|}
\hline Item & $\begin{array}{c}\text { Fator 1 } \\
\text { Qualidade da } \\
\text { habitação }\end{array}$ & $\begin{array}{c}\text { Fator } 2 \\
\text { Gestão doméstica }\end{array}$ & $\begin{array}{c}\text { Fator } 3 \\
\text { Rede e integração } \\
\text { social }\end{array}$ & $\begin{array}{c}\text { Fator } 4 \\
\text { Empregabilidade }\end{array}$ & Kappa a) \\
\hline 1 & 0,92 & & & & 0,83 \\
\hline 2 & 0,92 & & & & 0,78 \\
\hline 3 & 0,89 & & & & 0,82 \\
\hline 4 & 0,89 & & & & 0,70 \\
\hline 5 & & 0,36 & & 0,66 & 0,70 \\
\hline 6 & & 0,74 & & 0,35 & $0,92^{\mathrm{b})}$ \\
\hline 7 & & & 0,68 & 0,56 & 0,72 \\
\hline 8 & & & 0,65 & & $0,75^{\mathrm{b})}$ \\
\hline 9 & & & 0,34 & $-0,64$ & 0,71 \\
\hline 10 & & & 0,77 & & $0,64^{\mathrm{b})}$ \\
\hline 11 & 0,82 & & & & $0,73^{\text {b) }}$ \\
\hline 12 & & 0,89 & & & $\left.0,87^{\mathrm{b}}\right)$ \\
\hline 13 & & 0,90 & & & 0,62 \\
\hline $\begin{array}{l}\text { Variância } \\
\text { explicada }\end{array}$ & $32,43 \%$ & $16,88 \%$ & $12,32 \%$ & $8,94 \%$ & \\
\hline$\alpha$ Cronbach & 0,90 & 0,83 & 0,36 & 0,60 & \\
\hline $\begin{array}{c}\text { Total da } \\
\text { variância } \\
\text { explicada }\end{array}$ & $70,57 \%$ & & & & \\
\hline
\end{tabular}

Notas. São apresentadas apenas as cargas de saturação superiores a 0,30 . Os valores a negrito indicam o fator ao qual o item foi agregado na solução fatorial final adotada.

a) Para todos os valores de Kappa $\mathrm{p}<0,001$.

b) Para estes itens não foi possível calcular a estatística Kappa uma vez que não foi possível cumprir com o requisito de construir tabelas 2 vias simétricas para todos os valores da variável, para os dois avaliadores uma vez que alguns valores não foram igualmente observados para os dois avaliadores. São indicados os valores de correlação de Spearman. 
Regra geral, os três instrumentos avaliados apresentaram boas propriedades psicométricas que recomendam a sua utilização no contexto da realização de avaliações compreensivas e da investigação futura. A estrutura fatorial dos ISACM é teoricamente coerente e as diferentes dimensões apresentam bons índices de consistência interna e fidelidade entre avaliadores, o que pode contribuir para a qualidade das avaliações realizadas. Os resultados indicam, contudo, que pode ser pertinente a revisão dos itens na dimensão Rede e Integração Social do Instrumento de Síntese da Avaliação de Fatores Ambientais e Sociais. É possível que os profissionais estejam menos sensibilizados ou tenham menor preparação técnica para a avaliação destes itens. Pode acontecer que as metodologias de avaliação utilizadas para recolher a informação necessária para dar resposta a este item não tenham sido adequadas ou que os profissionais não disponham da informação necessária para cotar adequadamente os itens. De qualquer das formas estes itens merecem um estudo futuro mais aprofundado.

Em termos de validade concorrente, o Instrumento de Avaliação da Capacidade Parental parece apresentar, para várias dimensões, uma associação significativa com a previsão do risco de negligência e de abuso, no que diz respeito à contagem de comportamentos teoricamente considerados como de perigo. Os resultados oferecem alguns indicadores positivos sobre a validade dos ISACM.

Tabela 4. Distribuição dos participantes por nível de risco para a negligência e pela contagem do número de cotações de perigo

\begin{tabular}{|c|c|c|c|c|c|c|c|}
\hline \multirow[b]{2}{*}{$\begin{array}{l}\text { Nível de Risco } \\
\text { de negligência }\end{array}$} & \multicolumn{7}{|c|}{$\begin{array}{l}\text { Contagem de pontuações de perigo } \\
\text { (Soma de número de itens que pontuam } 1 \text { ou } 2 \text { ) }\end{array}$} \\
\hline & 0 & 1 & 2 & 3 & 4 & 5 & 6 \\
\hline & \multicolumn{7}{|c|}{ Prestação de Cuidados Básicos (7 itens) } \\
\hline Baixo & $30(78,9 \%)$ & $4(10,5 \%)$ & $1(2,6 \%)$ & $3(7,9 \%)$ & $0(0 \%)$ & $0(0 \%)$ & \\
\hline Moderado & $17(81,0 \%)$ & $2(9,5 \%)$ & $1(4,8 \%)$ & $0(0 \%)$ & $0(0 \%)$ & $1(4,8 \%)$ & \\
\hline Elevado & $9(69,2 \%)$ & $1(7,7 \%)$ & $1(7,7 \%)$ & $0(0 \%)$ & $1(7,7 \%)$ & $1(7,7 \%)$ & \\
\hline \multirow[t]{2}{*}{ Muito elevado } & $0(0 \%)$ & $0(0 \%)$ & $0(0 \%)$ & $0(0 \%)$ & $0(0 \%)$ & $1(100 \%)$ & \\
\hline & \multicolumn{7}{|c|}{ Segurança afetiva e supervisão (6 itens) } \\
\hline Baixo & $24(63,2 \%)$ & $8(21,1 \%)$ & $3(7,9 \%)$ & $2(5,3 \%)$ & $1(2,6 \%)$ & $0(0 \%)$ & $0(0 \%)$ \\
\hline Moderado & $14(66,7 \%)$ & $3(14,3 \%)$ & $2(9,5 \%)$ & $1(4,8 \%)$ & $0(0 \%)$ & $1(4,8 \%)$ & $0(0 \%)$ \\
\hline Elevado & $7(54,8 \%)$ & $1(7,7 \%)$ & $2(15,4 \%)$ & $1(7,7 \%)$ & $1(7,7 \%)$ & $1(7,7 \%)$ & $0(0 \%)$ \\
\hline \multirow[t]{2}{*}{ Muito elevado } & $0(0 \%)$ & $0(0 \%)$ & $0(0 \%)$ & $0(0 \%)$ & $0(0 \%)$ & $0(0 \%)$ & $1(100 \%)$ \\
\hline & \multicolumn{7}{|c|}{ Estimulação (3itens) } \\
\hline Baixo & $26(68,4 \%)$ & $8(21,1 \%)$ & $3(7,9 \%)$ & $1(2,6 \%)$ & & & \\
\hline Moderado & $16(76,2 \%)$ & $4(19,0 \%)$ & $0(0 \%)$ & $1(4,8 \%)$ & & & \\
\hline Elevado & $7(53,8 \%)$ & $5(38,5 \%)$ & $1(7,7 \%)$ & $0(0 \%)$ & & & \\
\hline \multirow[t]{2}{*}{ Muito elevado } & $0(0 \%)$ & $0(0 \%)$ & $0(0 \%)$ & $1(100 \%)$ & & & \\
\hline & \multicolumn{7}{|c|}{ Orientação e limites (3itens) } \\
\hline Baixo & $27(71,1 \%)$ & $4(10,5 \%)$ & $6(15,8 \%)$ & $1(2,6 \%)$ & & & \\
\hline Moderado & $18(85,7 \%)$ & $1(4,8 \%)$ & $1(4,8 \%)$ & $1(4,8 \%)$ & & & \\
\hline Elevado & $6(46,2 \%)$ & $4(30,8 \%)$ & $3(23,1 \%)$ & $0(0 \%)$ & & & \\
\hline \multirow[t]{2}{*}{ Muito elevado } & $0(0 \%)$ & $0(0 \%)$ & $0(0 \%)$ & $1(100 \%)$ & & & \\
\hline & \multicolumn{7}{|c|}{ Segurança Física (1 item) } \\
\hline Baixo & $34(89,5 \%)$ & $4(10,5 \%)$ & & & & & \\
\hline Moderado & $20(95,2 \%)$ & $1(4,8 \%)$ & & & & & \\
\hline Elevado & $11(84,6 \%)$ & $2(15,4 \%)$ & & & & & \\
\hline \multirow[t]{2}{*}{ Muito elevado } & $0(0 \%)$ & $1(100 \%)$ & & & & & \\
\hline & \multicolumn{7}{|c|}{ Proteção face a outros (1 item) } \\
\hline Baixo & $32(84,2 \%)$ & $6(15,8 \%)$ & & & & & \\
\hline Moderado & $19(90,5 \%)$ & $2(9,5 \%)$ & & & & & \\
\hline Elevado & $10(76,9 \%)$ & $3(23,1 \%)$ & & & & & \\
\hline Muito elevado & $0(0 \%)$ & $1(100 \%)$ & & & & & \\
\hline
\end{tabular}


No entanto, e muito embora os níveis de risco inferiores tendam a estar associados a um número maior de casos sem registo de indicadores de perigo ao nível da capacidade parental, os resultados dificilmente permitem que se façam inferências lineares, a partir dos Instrumentos de Síntese, para a probabilidade de ocorrência de negligência ou abuso. Não obstante, e muito embora não possa constituir critério único de avaliação, a ausência de cotações em várias dimensões de perigo no Instrumento de Síntese da Avaliação Parental pode estar associada com uma menor probabilidade de ocorrência daqueles problemas, considerando-se a estimativa do risco efetuada na CFRA. Nos níveis superiores parece ser mais difícil diferenciar-se o nível de risco por número de comportamentos de perigo com exceção do nível muito elevado em que o único caso registado para a negligência apresentou, tendencialmente a pontuação máxima nos ISACM o mesmo não se verificando, contudo, para o risco de abuso. Há que referir que apenas se compararam indicadores de perigo ao nível da capacidade parental e que o CFRA inclui itens relacionados não só com a ocorrência passada do abuso mas também com fatores relacionados com características dos próprios pais e do funcionamento familiar, o que podem contribuir para explicar parcialmente os resultados.

Tabela 5. Distribuição dos participantes por nível de risco para o abuso e pela contagem do número de cotações de perigo

Contagem de pontuações de perigo

(Soma de número de itens que pontuam 1 ou 2)

\begin{tabular}{|c|c|c|c|c|c|c|c|}
\hline $\begin{array}{l}\text { Nível de Risco } \\
\text { de abuso }\end{array}$ & 0 & 1 & 2 & 3 & 4 & 5 & 6 \\
\hline & \multicolumn{6}{|c|}{ Prestação de Cuidados Básicos (7 itens) } & \\
\hline Baixo & $30(81,1 \%)$ & $4(10,8 \%)$ & $1(2,7 \%)$ & $1(2,7 \%)$ & $0(0 \%)$ & $1(2,7 \%)$ & \\
\hline Moderado & $14(73,7 \%)$ & $2(10,5 \%)$ & $0(0 \%)$ & $2(10,5 \%)$ & $0(0 \%)$ & $1(5,3 \%)$ & \\
\hline Elevado & $9(81,8 \%)$ & $0(0 \%)$ & $1(9,1 \%)$ & $0(0 \%)$ & $1(9,1 \%)$ & $0(0 \%)$ & \\
\hline \multirow[t]{2}{*}{ Muito elevado } & $2(40,0 \%)$ & $1(20 \%)$ & $1(20 . \%)$ & $0(0 \%)$ & $0(0 \%)$ & $1(20 \%)$ & \\
\hline & \multicolumn{6}{|c|}{ Segurança afetiva e supervisão (6 itens) } & \\
\hline Baixo & $26(70,3 \%)$ & $6(16,2 \%)$ & $4(10,8 \%)$ & $1(2,7 \%)$ & $0(0 \%)$ & $0(0 \%)$ & \\
\hline Moderado & $10(52,6 \%)$ & $6(26,3 \%)$ & $2(10,5 \%)$ & $1(5,3 \%)$ & $1(5,3 \%)$ & $0(0 \%)$ & \\
\hline Elevado & $7(15,9 \%)$ & $1(9,1 \%)$ & $0(0 \%)$ & $2(18,2 \%)$ & $0(0 \%)$ & $1(9,1 \%)$ & \\
\hline \multirow[t]{2}{*}{ Muito elevado } & $1(20 \%)$ & $0(0 \%)$ & $1(20 \%)$ & $0(0 \%)$ & $1(20 \%)$ & $1(20 \%)$ & \\
\hline & \multicolumn{6}{|c|}{ Estimulação (3itens) } & \\
\hline Baixo & $28(75,7 \%)$ & $8(21,6 \%)$ & $0(0 \%)$ & $1(2,7 \%)$ & & & \\
\hline Moderado & $13(68,4 \%)$ & $5(26,3 \%)$ & $1(5,3 \%)$ & $0(0 \%)$ & & & \\
\hline Elevado & $7(63,6 \%)$ & $2(18,2 \%)$ & $2(18,2 \%)$ & $0(0 \%)$ & & & \\
\hline \multirow[t]{2}{*}{ Muito elevado } & $0(0 \%)$ & $2(40 \%)$ & $1(20 \%)$ & $2(40 \%)$ & & & \\
\hline & \multicolumn{6}{|c|}{ Orientação e limites (3itens) } & \\
\hline Baixo & $29(28,4 \%)$ & $4(10,8 \%)$ & $4(10,8 \%)$ & $0(0 \%)$ & & & \\
\hline Moderado & $12(63,2 \%)$ & $4(21,1 \%)$ & $2(10,5 \%)$ & $1(5,3 \%)$ & & & \\
\hline Elevado & $8(72,7 \%)$ & $1(9,1 \%)$ & $2(18,2 \%)$ & $0(0 \%)$ & & & \\
\hline \multirow[t]{2}{*}{ Muito elevado } & $1(20 \%)$ & $0(0 \%)$ & $2(40 \%)$ & $2(29 \%)$ & & & \\
\hline & \multicolumn{6}{|c|}{ Segurança Física (1 item) } & \\
\hline Baixo & $34(91,9 \%)$ & $3(8,1 \%)$ & & & & & \\
\hline Moderado & $17(89,5 \%)$ & $2(10,5 \%)$ & & & & & \\
\hline Elevado & $10(90,9 \%)$ & $1(9,1 \%)$ & & & & & \\
\hline \multirow[t]{2}{*}{ Muito elevado } & $3(60 \%)$ & $2(40 \%)$ & & & & & \\
\hline & \multicolumn{6}{|c|}{ Proteção face a outros (1 item) } & \\
\hline Baixo & $34(91,9 \%)$ & $3(8,1 \%)$ & & & & & \\
\hline Moderado & $14(73,7 \%)$ & $5(26,3 \%)$ & & & & & \\
\hline Elevado & $11(100 \%)$ & $0(0 \%)$ & & & & & \\
\hline Muito elevado & $1(20 \%)$ & $4(80 \%)$ & & & & & \\
\hline
\end{tabular}


Entre as limitações associadas a este estudo incluem-se o reduzido tamanho da amostra e o fato de apenas terem sido incluídos participantes sinalizados para serviços de apoio familiar pela vivência de problemas de ordem psicossocial e, na maioria dos casos, situações de perigo ou risco psicossocial. Seria relevante realizar, novamente, este estudo, no futuro, com uma amostra da comunidade em geral comparada com uma amostra acompanhada por serviços. O Instrumento de Síntese da Avaliação da Capacidade Parental foi comparado com um instrumento de avaliação do risco que não foi ainda avaliado para a população portuguesa do ponto de vista da sua validade preditiva pelo que a interpretação dos resultados deve ser cautelosa. Por outro lado, a seleção dos pontos 1 e 2 como correspondendo a situações de perigo não tem sustentação empírica. A investigação futura deve replicar o estudo com diferentes amostras e avaliar pontos de corte que permitam a identificação de situações de perigo e de risco psicossocial.

\section{Referências}

Abbad, G. S., Queiroga, F., \& Zerbini, T. (2012). Fundamentos teóricos e metodológicos em análise de instrumentos. In G. S., Abbad, L. Mourão, P. P. M. Meneses, T. Zerbini, J. E. BorgesAndrade \& R. Vilas-Boas (Eds.), Métodos para avaliação em treinamento, desenvolvimento e educação: Ferramentas para gestão de pessoas (pp. 64-76). Porto Alegre: Art med.

Azar, S. T., Lauretti, A. F., \& Loding, B. V. (1998). The evaluation of parental fitnesss in termination of parental rights cases: A functional-contextual perspective. Clinical Child and Family Psychology Review, 1(2),77- 100. doi: 10.1023/A:1021883611965

Belsky, J. (1980). Child maltreatment: An ecological integration. American Psychologist, 35(4), 320-335. doi: 10.1037/0003066X.35.4.320

Belsky, J. (1984). The determinants of parenting. A process model. Child Development, 55(1), 83-96.

Budd, K. S. (2001). Assessing parenting competence in child protection cases: A clinical practice model. Clinical Child and Family Psychology Review, 4(1), 1-18. doi: 10.1023/A:1009548509598

Ciccheti D., \& Valentino, K. (2006). An ecological-transactional perspective on child maltreatment: Failure of the average expectable environmental and its influence in child development. In D. Cicchetti \& D. J. Cohen (Eds.), Developmental sychopathology, Vol. 3 (2nd ed.) (pp. 129-199). New York: John Wiley \& Sons.

Cicchetti, D., \& Lynch, M. (1995). Failures in the expectable environment and their impact on individual development: The case of child maltreatment. In D. Cicchetti \& D. Cohen (Eds.), Developmental Psychopathology. Vol. 2 Risk, disorder and adaptation (pp. 32-71). New York : John Wiley and Sons.

Cicchetti, D., \& Cohen, D. J. (Eds.) (2006). Developmental psychopathology, Vol. 3 (2nd ed.). New York: John Wiley \& Sons.
Cicchetti, D., \& Toth, S. L. (1997). Transactional ecological systems in developmental psychopathology. In S. S. Luthar, J. A. Barack, D. Cicchetti \& J. R. Weisz (Eds.), Developmental psychopathology: Perspectives on adjustment, risk and disorder (pp. 317-349). Cambridge: Cambridge University Press.

Comissão Nacional de Protecção de Crianças e Jovens em Risco [CNPCJR] (2009). Relatório anual de avaliação das actividades das comissões de protecção de crianças e jovens. Lisboa: Comissão Nacional de Protecção de Crianças e Jovens em Risco.

Corcoran, J., \& Nichols-Casebolt, A. (2004). Risk and resilience ecological framework for assessment. Child and Adolescent Social Work Journal, 21(3), 211-235. doi: 10.1023/B:CA SW.0000028453.79719.65

Cummings, E. M., Davies, P. T., \& Campbell, S. B. (2000). Developmental psychopathology and family process. Theory, research, and clinical implications. New York: The Guilford Press.

Darlington, Y., Healy, K., \& Feeney, J. A. (2010). Approaches to assessment and intervention across four types of child and family welfare services. Children and Youth Services Review, 32(3), 356-364. doi:10.1016/j.childyouth.2009.10.005

DePanfilis, D. (1996). Implementing child mistreatment risk assessment systems: Lesson from theory. Administration in Social Work, 20(2), 41-59. doi: 10.1300/J147v20n02_04

Department of Health (2000). Framework for the assessment of children in need and their families. London: Stationery Office.

Farnfield, S. (2008). A theoretical model for the comprehensive assessment of parenting. British Journal of Social Work, 38(6), 1076-1099. doi: 10.1093/bjsw/bcl395

Fowler, J. (2003). A practitioner's tool for child protection and the assessment of parents. London: Jessica Kingsley Publishers.

Jack, G. (2001). Ecological perspectives in assessing children and families. In J. Horwath (Ed.), The child's world. Assessing children in need (pp. 53-74). London: Jessica Kingsley Publishers.

Johnson, K. \& Wagner, D. (2003). California Structured Decision Making TM. Risk Assessment revalidation: A prospective study. Children's Research Center. Retrieved from http:/www.nccdcrc.org/crc/crc/pubs/CACPS2003RiskValidationReport.pdf

Johnson, M. A., Stone, S., Lou, C., Vu, C., Ling, J., Mizhari, P., \& Austin, M. J. (2006). Family assessment in child welfare services: Instrument comparisons. Retrieved from http://www. childwelfare.gov/famcentered/casework/assessment.cfm

Johnson, W. L. (2011). The validity and utility of the California Family Risk Assessment under practice conditions in the field: A prospective study. Child Abuse \& Neglect, 35(1), 18-28. doi: 10.1016/j.chiabu.2010.08.002

Jones, D. (2001). The assessment of parental capacity. In J. Horwath (Ed.), The child's world. Assessing children in need (pp. 255272). London: Jessica Kingsley Publishers.

Landis, J. R., \& Koch, G. G. (1977). The measurement of observer agreement for categorical data. Biometric, 33, 159-174.

Laros, J. A. (2005). O uso da análise fatorial: Algumas diretrizes para pesquisadores. In Pasquali. L. (Ed.), Análise fatorial para pesquisadores (pp. 141-160). Brasília: LabPAM Saber e Tecnologia. 
Léveillé, S., \& Chamberland, C. (2010). Toward a general model for child welfare and protection services: A meta-evaluation of international experiences regarding the adoption of the Framework for the Assessment of Children in Need and Their Families (FACNF). Children and Youth Services Review, 32, 929-944.

Masten, A. S. (2007). Resilience in developing systems: Progress and promise as the fourth wave rises. Development and Psychopathology, 19(3), 921-930. doi: 10.1017/ S0954579407000442

Melo, A. T., \& Alarcão, M. (2008). Manual de preenchimento dos Instrumentos de Síntese da Avaliação Compreensiva no MAIFI. Disponível com os autores.

Reder, P., \& Lucey, C. (Eds.). (1995). Assessment of parenting. Psychiatric and psychological contributions. Hove: BrunnerRoutledge.

Rose, W. (2001). Assessing children in need and their families. In J. Horwath (Ed.), The child's world. Assessing children in need (pp. 35-49). London: Jessica Kingsley Publishers.

Schene, P. (2005). Comprehensive family assessment guidelines for child welfare. National child welfare resource center for familycentered practice. Retrieved from http://www.childwelfare. gov/famcentered/casework/assessment.cfm
Spratt, T., \& Devaney, J. (2009). Identifying families with multiple problems: Perspectives of practitioners and managers in three nations. British Journal of Social Work, 39(3), 418-434. doi: $10.1093 / \mathrm{bjsw} / \mathrm{bcm} 150$

Torres, A. (Coord.) (2008). Estudo de diagnóstico e de avaliação das comissões de protecção de crianças e jovens. Lisboa: Centro de Investigação e Estudos de Sociologia, Instituto Superior de Ciências do Trabalho e da Empresa.

Wagner, D. \& Johnson, K. (1999). 13th national rountable on CPS risk assessment. Using actuarial risk assessment to target service interventions in pilot California counties. Madison, Wisconsin: Children's Research Center. Retrieved from http:// www.nccd-crc.org/crc/crc/pubs/13th_roundtable_ca_risk.pdf

Wald, M. S., \& Woolverton, M. (1990). Risk assessment: The emperor's new clothes? Child Welfare, LXIX, 6, 483-511.

Walsh, F. (2002). A family resilience framework: Innovative practice applications. Family Relations, 51(2), 130-137. doi: 10.1111/j.1741-3729.2002.00130.x

Wise, S. (2003). The child in family services. Expanding child abuse prevention. Australian Social Work, 56(3),183-195. doi: 10.1046/j.0312-407x.2003.00081.x

Recebido em 06.09.2011 Primeira decisão editorial em 17.06.2013 Versão final em 13.07.2013 Aceito em 01.08.2013 
Apêndice 1. Listagem de itens do Instrumento de Síntese da Avaliação da Capacidade Parental no MAIFI

Itens do Instrumento de Síntese da Avaliação da Capacidade Parental

Item1- Capacidade de proporcionar alimentos adequados às necessidades nutricionais da criança quer em quantidade quer em qualidade

Item 2- Capacidade de garantir higiene na confecção alimentar e qualidade dos alimentos

Item 3- Capacidade de acompanhar/supervisionar as refeições das crianças

Item 4- Capacidade de proporcionar banhos regulares e higiene cuidada de todas as zonas do corpo

Item 5- Capacidade de utilizar utensílios e produtos de higiene adequados limpos e em bom estado de conservação e manter limpos os espaços de realização de cuidados de higiene

Item 6- Capacidade de garantir tratamento adequado de roupas, proporcionar vestuário próprio à criança, adequado ao clima, em boas condições

Item 7- Capacidade de garantir o cumprimento com indicações médicas e acompanhamento regular de médico assistente/de família e vacinação obrigatória

Item 8- Capacidade de criar condições para garantir a segurança da criança relativamente à proteção perante objetos, substâncias ou circunstâncias que ameacem a segurança física da criança (deixar fora do acesso da criança objetos cortantes ou tóxicos, impedir acesso a janelas ou varandas, circuitos elétricos, etc.)

Item 9- Capacidade de proteção da criança face a contatos com adultos ou outras crianças passíveis de colocarem em causa a sua segurança

Item 10- Capacidade de supervisionar e monitorizar o comportamento da criança

Item 11- Capacidade de estar atento, alerta e sensível, de perceber e interpretar adequadamente o comportamento, os estados emocionais e as necessidades da criança

Item 12- Capacidade de ser responsivo ao comportamento e perturbação emocional da criança; proporcionar, conforto, calor e cuidado nas respostas à criança, de forma previsível e consistente

Item 13- Capacidade de aceitar incondicionalmente a criança e de a apreciar, de proporcionar atenção positiva, elogios e reforços positivos.

Item 14- Capacidade de proporcionar segurança, apoiar e incentivar a exploração do mundo físico e relacional por parte da criança de forma que promova autonomia e sentido de competência da criança

Item 15- Capacidade de manter expectativas desenvolvimentalmente apropriadas para o comportamento da criança

Item 16- Capacidade de garantir o envolvimento da criança e atividades ou experiências passíveis de promoverem e estimularem o seu desenvolvimento a diferentes níveis (físico, psicológico, social, cognitivo, emocional, académico)

Item 17- Capacidade de estabelecer regras, tarefas e limites adequados

Item 18- Capacidade de utilizar estratégias de disciplina positivas e consistentes

Item 19- Capacidade de promover a competência emocional da criança

Item 20- Capacidade de assegurar estabilidade no acompanhamento da vida da criança e de manter estabilidade nos contextos de vida da criança.

Item 21- Capacidade de proporcionar oportunidades para que criança crie vínculos com várias figuras e contextos importantes e que mantenha contatos regulares com as mesmas. 
Apêndice 2. Listagem de itens dos Instrumentos de Síntese da Avaliação Compreensiva no MAIFI (cont.)

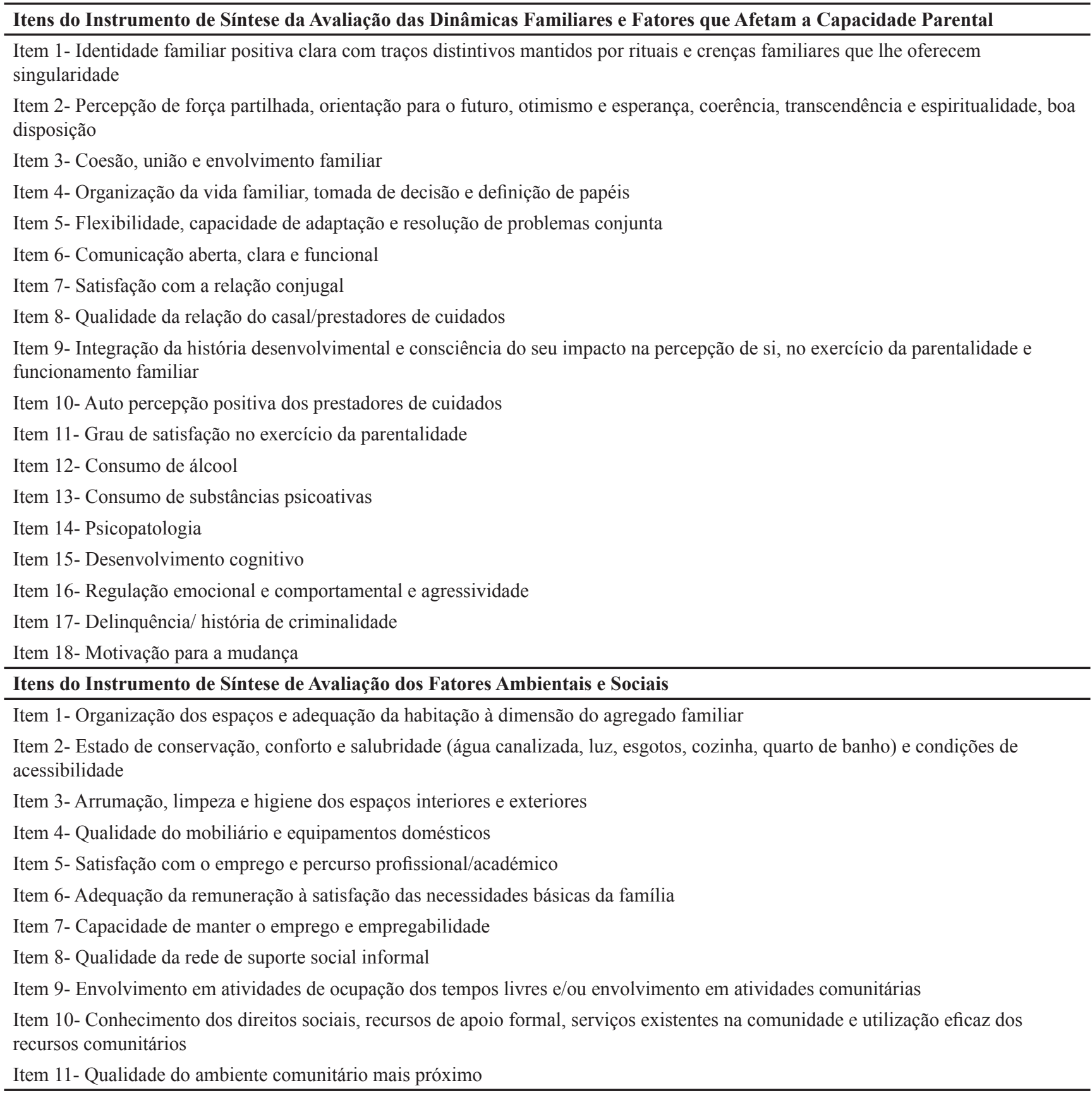

